\title{
Effect of soil moisture on microwave scattering for remote sensing
}

\section{SINGH}

Department of Physics, Government Science Autonomous Post Graduate College, Bilaspur 495006, India

MS received 24 March 1998; revised 15 May 1999

\begin{abstract}
The main objective of remote sensing is to design space borne microwave sensors to sense a target and derive useful geophysical parameters. For this purpose, the knowledge of the target characteristics must be obtained through ground based remote sensing. In this paper, soil has been taken as the target. Various percentages of gravimetric soil moisture $\left(m_{\mathrm{g}}\right)$ have been taken for establishing its relation with the scattering coefficient $\left(\sigma_{0}\right)$ for both like polarizations at X-band frequencies. A linear model has been developed for correlating these two variables (i.e. $m_{\mathrm{g}}$ and $\sigma_{0}$ ). Using this model, regression analysis has been done for obtaining different regression parameters and predicted values. Treating measurements from bare smooth soil fields with different soil moistures, the data were analysed to examine the effect of soil moisture on scattering coefficient $\left(\sigma_{0}\right)$ at $9.50 \mathrm{GHz}$ frequency in X-band. The scattering coefficient increases with increase in soil moisture content. Different regression parameters have been obtained, which show that the best look-angle is at $25^{\circ}$ for $\mathrm{HH}$-pol and $60^{\circ}$ for VV-pol for observing $\sigma_{0}$ from bare moist soil. Data analysis indicated that the basic cause and effect relationship between the sensor measurements and soil moisture can be extrapolated from theory and small-scale tests to larger resolution elements observed by the sensing aircraft. These results indirectly provide reference data for a satellite-borne remote sensor. From the results, the look angles suitable for operation with radar antennas can be suggested.
\end{abstract}

Keywords. Remote sensing; microwave scattering; soil moisture; scattering coefficient.

\section{Introduction}

Forecasts related to hydrological and agricultural water management decisions can be improved by timely and accurate observations of soil moisture. However, conventional measurements are cumbersome and impractical, especially when frequent observations over large regions are required. Remote sensing techniques are ideal for such purposes and are being evaluated for increasing the precision in soil moisture sensing. Microwave systems appear to be the best suited for potential remote sensing applications because they 
provide a direct estimate of soil moisture. They are extendable to satellite platforms and are weather independent. The principal advantage of remote sensing, as opposed to conventional data collection methods, is that spatially distributed and frequent observation of a phenomenon can be made rapidly over a large area. Microwave remote sensing holds great promise for hydrology because the primary physical property that affects the measurement is directly dependent on the amount of water present.

The dielectric properties of the target are some of the principal determinants of microwave-scattering coefficients. A soil target consists of air, water and varieties of soil constituents. At microwave frequencies, the real part of the complex numbers representing the dielectric constant are air, $K=1$, soil, $K=4$ and water, $K=80$. These large contrasts in $K$ make it possible to measure soil moisture.

Several researchers have used active microwave sensors in order to (i) test the capability of such equipment to measure the soil surface water content (Ulaby et al 1978; Le Toan et al 1981; Jackson \& O'Neill 1985), and (ii) monitor the water budget at a field or regional scale (Bernard et al 1981; Pervot et al 1984). Consequently, to retrieve soil moisture from a radar scattering coefficient $\left(\sigma_{0}\right)$ implies establishing relationships between the gravimetric water content $\left(m_{\mathrm{g}}\right)$ and $\sigma_{0}$ for all available combinations of look-angle. To fulfil the aim of the air-borne or space-borne mission using active microwave remote sensing for land applications, there is an urgent need for the development of numerical procedures comparable with existing technology which may be implemented to retrieve soil moisture information from remote data.

In this paper, an attempt has been made to highlight the effect of soil moisture on scattering coefficients for microwave response. For this purpose, we have developed a simple linear model with large sets of data $\left(m_{\mathrm{g}}, \sigma_{0}\right)$ and have established relationships for scattering coefficient with selected sets of look-angle $(\theta)$ for the X-band at different soil moistures.

\section{Experimental technique}

A bistatic scatterometer system is used for observing moist soil. Schematic representation of the bistatic scatterometer system employed for outdoor microwave remote sensing experiments is shown in figure 1. The system consists of two pyramidal horn antennas in which one was connected to the transmitter and the other to the receiver having half-power beam width equal to $23.5^{\circ}$ in $\mathrm{H}$-plane and $24^{\circ}$ in E-plane, with a gain of $20.74 \mathrm{~dB}$ and $20.27 \mathrm{~dB}$ respectively and operated at $9.5 \mathrm{GHz}$. These antennas were mounted on wooden portable stands. The system had a facility to vary the height and the look-angle.

The polarization of the radiated signal was also changed by using $90^{\circ} \mathrm{E}-\mathrm{H}$ twist. For each degree variation of look-angle, a particular height of the antenna and the separation of the antenna from the centre of the target were calculated. This technique helped us to calculate the far-field and near-field effects. The whole system was calibrated by noting the signal returned from a flat aluminium sheet placed between the transmitting and receiving antennas. The standard technique is discussed in detail by Singh \& Jha (1987).

\section{Theoretical approach}

When electromagnetic waves are incident on a terrain, their reflection/scattering depends basically on two factors: (i) target parameters, and (ii) system parameters, where 


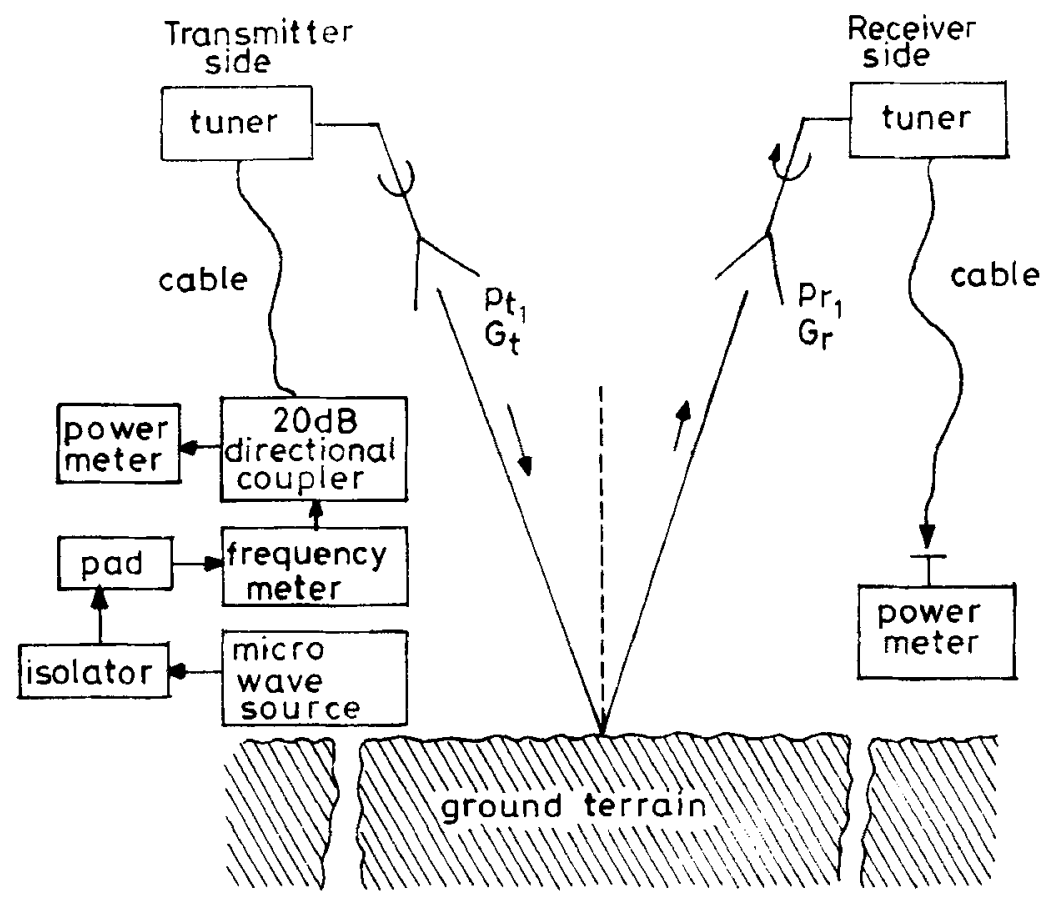

Figure 1. Schematic diagram of bistatic scatterometer system.

the target parameters contain the surface geometry and subsurface characteristics of the target including complex dielectric properties and the system parameters include the properties of incident waves, i.e., wavelength, like- or cross-polarization and look-angle $\theta$. The dielectric properties for soil are strongly dependent uponi the amount of water/moisture in it as well as its texture and thus the amount of water/moisture added to dry soil increases the magnitude of its dielectric constant as well as its conductivity, which in turn affects the scattering and emission of electromagnetic waves.

The scattering coefficient $\left(\sigma_{0}\right)$ of the target has been computed using the radar equation given by Singh \& Jha (1987).

$$
\sigma_{0}=R^{1}\left|R_{0}\right|^{2} / I_{0}
$$

where, $R_{0}$ is the reflection coefficient, $\sigma_{0}$ is scattering coefficient, $R^{1}$ is the distance of the transmitting and receiving antennas from the centre of the illuminated area and $I_{0}$ is the illuminated area of the target.

In (1), $\sigma_{0}$ is directly related to reflectivity, whereas reflectivity depends upon target characteristics (i.e. dielectric constant of soil). Therefore $\sigma_{0}$ is directly related to soil moisture and can be related to a general form

$$
\sigma_{0}=\frac{\mathrm{d} \sigma_{0}(\theta, p)}{\mathrm{d}\left(m_{\mathrm{g}}\right)} \times m_{\mathrm{g}}+K_{\mathrm{l}},
$$

where, $m_{\mathrm{g}}$ is gravimetric soil moisture, $\mathrm{d} \sigma_{0}(\theta, p) / \mathrm{d}\left(m_{\mathrm{g}}\right)$ is slope and $K_{\mathrm{I}}$ is a constant, which depends upon the target characteristics and sensor parameters. 


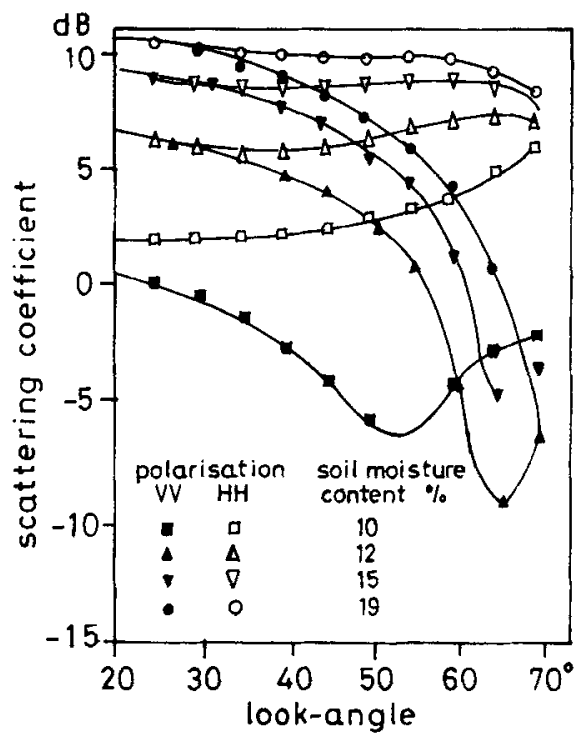

Figure 2. Angular variation of scattering coefficient as a function of soil moisture by weight (gravimetric) for VV- and $\mathrm{HH}$-polarizations at $9.5 \mathrm{GHz}$, temp. $=23^{\circ} \mathrm{C}$ and surface roughness $=0.4 \mathrm{~cm}$.

\section{Results and discussion}

The soil surface from which the data were obtained had $6 \%$ gravel, $55 \%$ sand, $35 \%$ silt and $4 \%$ clay. The ground surface was taken as smooth (as the roughness parameter was equal to $0.4 \mathrm{~cm}$ ) and the soil moisture content had varying values. Figure 2 shows the angular variation of scattering coefficient for bare smooth moist soil at various moisture contents for VV- and $\mathrm{HH}$-polarisations respectively.

The curves plotted for VV-polarization show that different soil moisture contents $(10,12,15$ and $19 \%)$ give $20 \mathrm{~dB}$ variations of scattering coefficient for the observed range of look-angles i.e. $20^{\circ}$ to $70^{\circ}$. The highest percentage of gravimetric soil moisture gives maximum scattering coefficient. From the curves plotted for HH-polarization it is seen that the behaviour of the curve is approximately opposite to the VV-polarization. The variation of scattering coefficient is approximately $10 \mathrm{~dB}$ for HH-polarization. In this case also, the maximum scattering coefficient is at the highest soil moisture content (i.e. $19 \%$ ).

The variations in percentage of gravimetric soil moisture $\left(m_{\mathrm{g}}\right)$ with scattering coefficient measured in the top 5-centimetre layer for look-angles $60^{\circ}$ and $25^{\circ}$ for VV-pol and HH-pol respectively at $\mathrm{X}$-band $(9.5 \mathrm{GHz})$ are shown in figures 3 and 4 . In both the figures, it is observed that the scattering coefficient increases as the percentage of soil moisture increases. Similar results have been obtained by Ulaby et al (1982), Dobson \& Ulaby (1981) and Bertuzzi et al (1992) for C- and L-bands. These results at the X-band complement earlier research efforts at other bands. For the X-band, the intercept changes as compared to those for the C- and L-bands.

The slope obtained in our case for the X-band is different from those reported at both the C- and L-bands, given by Bradley \& Ulaby (1980). For the sake of comparison, the equations of regression lines given by Schmugge et al (1980) are compared with our 


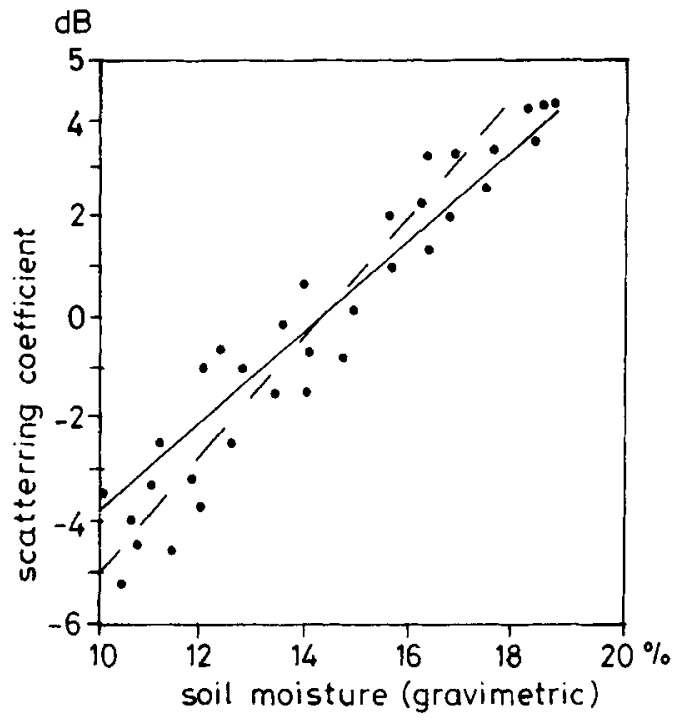

Figure 3. Variation of scattering coefficient with percentage of soil moisture (gravimetric) for VV-pol. Frequency $=9.5 \mathrm{GHz}$, look-angle $=60^{\circ}, \sigma_{0}=-5.356+$ $0.96 m_{\mathrm{g}}$, regression line.

$\mathrm{X}$-band as below:

$$
\sigma_{0}=-10.9+0.33 m_{\mathrm{v}},
$$

for C-band $\left(4.75 \mathrm{GHz}, \mathrm{HH}\right.$-pol, $15^{\circ}$ ),

$$
\sigma_{0}=-15.5+0.35 m_{\mathrm{v}},
$$

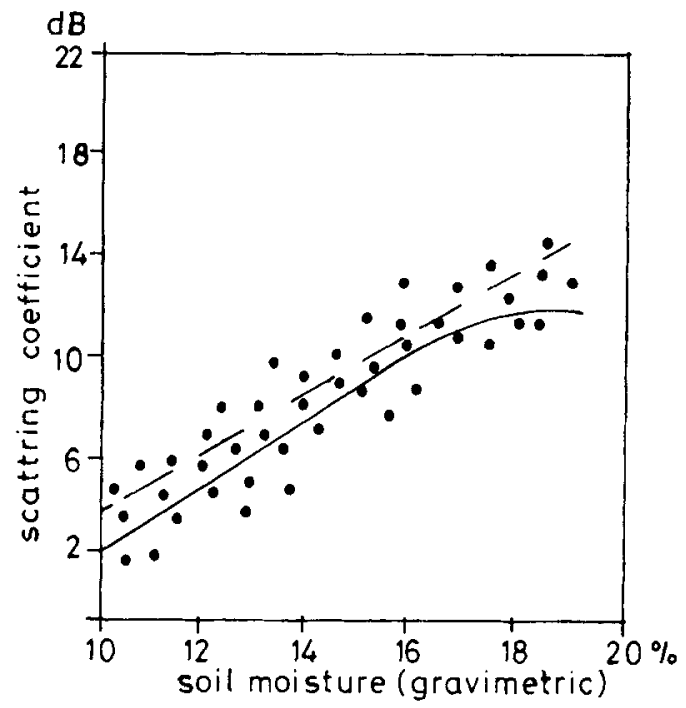

Figure 4. Variation of scattering coefficient with percentage of soil moisture (gravimetric) for $\mathrm{HH}$-pol. Frequency $=9.5 \mathrm{GHz}$, look-angle $=25^{\circ}, \sigma_{0}=-2.984+$ $0.683 m_{\mathrm{g}}, \ldots \ldots \ldots . .$. regression line. 
for L-band (1.6 GHz, HH-pol, $\left.15^{\circ}\right)$,

$$
\sigma_{0}=-2.984+0.683 m_{\mathrm{g}}
$$

for X-band $\left(9.5 \mathrm{GHz}, \mathrm{HH}\right.$-pol, $\left.25^{\circ}\right)$.

Comparing (5) with (3) and (4), it is inferred that the slope of the X-band is greater because gravimetric soil moisture has been considered here, instead of the volumetric soil moisture taken by others. It also depends upon frequency. Similarly, the equation for VVpol for the $\mathrm{X}$-band at $60^{\circ}$ is

$$
\sigma_{0}=5.356+0.96 m_{\mathrm{g}} \text {. }
$$

Now, comparing (5) and (6) for HH- and VV-pol, it is observed that the slope is larger for VV-pol than for HH-pol. The slope defines the sensitivity of the scattering coefficient to the soil moisture. It is found that the sensitivity to soil moisture is better with VV-than with HH-pol.

Tables 1 and 2 show various regression parameters. The slope of the regression line, which shows the sensitivity of the relation between scattering coefficient and soil moisture, decreases with the look-angle for both HH-pol and VV-pol, and the constants of the intercept increase as the look-angle increases. The intercept refers to attenuation. The most suitable result for the regression line is found at look-angle $25^{\circ}$ for $\mathrm{HH}$-pol and $60^{\circ}$ for VVpol. From the same table, it is observed that variation in correlation coefficient $(r)$ changes with look-angle, which shows the strength of the relationship between $\sigma_{0}$ and $m_{\mathrm{g}}$. It is found that, for $\mathrm{HH}$-pol, $r$ continuously decreases with respect to $\theta$ while $r$ decreases up to $50^{\circ}$ and then increases for $\mathrm{VV}$-pol. The best value of $r$ for $\mathrm{HH}$-pol is 0.842 at look-angles of $25^{\circ}$ and 0.95 for $V V$-pol at $60^{\circ}$. However the $60^{\circ}$ angle is not preferred due to problems in radar remote sensing. It is clear that the VV-pol gives much better performance in comparison to $\mathrm{HH}$-pol as expected.

Figures 5 and 6 show the correlation between observed and calculated values. These values are best-fitted at look-angles $25^{\circ}$ and $60^{\circ}$ for $\mathrm{HH}$-pol and VV-pol, respectively. Tables 1 and 2 also show the angular variation of coefficient of determination $\left(r^{2}\right)$ which shows the dependence of scattering coefficient on soil moisture. The maximum value of $r^{2}$ is 0.71 at look-angle $25^{\circ}$ and, with further increase in the value of look-angle,

Table 1. Linear regression results of scattering coefficient vs. gravimetric soil moisture at different look-angles for HH-pol.

\begin{tabular}{lcccccc}
\hline Angle (deg.) & Slope & Intercept & $r^{2}$ & $r$ & SE* & SE of estimate \\
\hline 20 & 0.696 & 2.182 & 0.682 & 0.826 & 0.168 & 1.515 \\
25 & 0.683 & 2.984 & 0.710 & 0.842 & 0.154 & 1.391 \\
30 & 0.620 & 6.620 & 0.637 & 0.789 & 0.166 & 1.493 \\
35 & 0.565 & 8.809 & 0.554 & 0.744 & 0.179 & 1.616 \\
40 & 0.513 & 10.018 & 0.449 & 0.706 & 0.182 & 1.639 \\
45 & 0.521 & 12.178 & 0.489 & 0.699 & 0.188 & 1.695 \\
50 & 0.461 & 13.575 & 0.440 & 0.664 & 0.184 & 1.657 \\
55 & 0.431 & 13.555 & 0.447 & 0.669 & 0.169 & 1.526 \\
60 & 0.372 & 14.929 & 0.283 & 0.619 & 0.167 & 1.503 \\
65 & 0.205 & 15.715 & 0.149 & 0.385 & 0.174 & 1.566 \\
70 & 0.130 & 16.003 & 0.127 & 0.356 & 0.178 & 1.820 \\
\hline
\end{tabular}

* SE -- standard error 
Table 2. Linear regression results of scattering coefficient vs. gravimetric soil moisture at different look-angles for VV-pol.

\begin{tabular}{lcrcccc}
\hline Angle (deg.) & Slope & Intercept & $r^{2}$ & $r$ & SE & SE of estimate \\
\hline 20 & 1.11 & -8.90 & 0.81 & 0.90 & 0.18 & 1.68 \\
25 & 1.10 & -9.90 & 0.82 & 0.906 & 0.37 & 2.51 \\
30 & 1.12 & -9.75 & 0.79 & 0.89 & 0.40 & 2.71 \\
35 & 1.10 & -10.00 & 0.77 & 0.877 & 0.42 & 2.87 \\
40 & 1.17 & -11.82 & 0.76 & 0.872 & 0.45 & 3.10 \\
45 & 1.25 & -13.85 & 0.74 & 0.86 & 0.51 & 3.49 \\
50 & 1.27 & -15.33 & 0.71 & 0.84 & 0.56 & 3.81 \\
55 & 0.95 & -15.23 & 0.81 & 0.90 & 0.34 & 2.18 \\
60 & 0.96 & -5.35 & 0.91 & 0.95 & 0.20 & 1.41 \\
65 & 0.85 & -1.42 & 0.43 & 0.65 & 0.28 & 1.85 \\
70 & 0.33 & -0.42 & 0.37 & 0.61 & 0.30 & 2.04 \\
\hline
\end{tabular}

the $r^{2}$ value decreases for HH-pol. Similarly, for VV-pol the maximum value of $r^{2}$ is 0.918 at $60^{\circ}$.

In these tables, the angular variation of standard error (SE) of scattering coefficient is also shown. The value of SE varies throughout. Standard error provides an idea about the unreliability of a sample. The greater the standard error, the greater the departure of the actual value from that expected and hence the greater is the degree of unreliability in the measurements. The SE also determines the limit within which the scattering coefficient and soil moisture values are expected to lie. The minimum value of $\mathrm{SE}$ is 0.154 at $25^{\circ}$ and 0.20 at $60^{\circ}$ for $\mathrm{HH}$ - and VV-pol respectively. It means that the SE of the scattering coefficient depends upon look-angle and the best look-angles are $25^{\circ}$ and $60^{\circ}$ for $\mathrm{HH}$-pol and VV-pol respectively for observing bare smooth soil moisture.

\section{Conclusio :}

In this paper an attempt has been made to correlate the relationship between soil moisture and scattering coefficient. This can be done by using a linear regression equation to explain

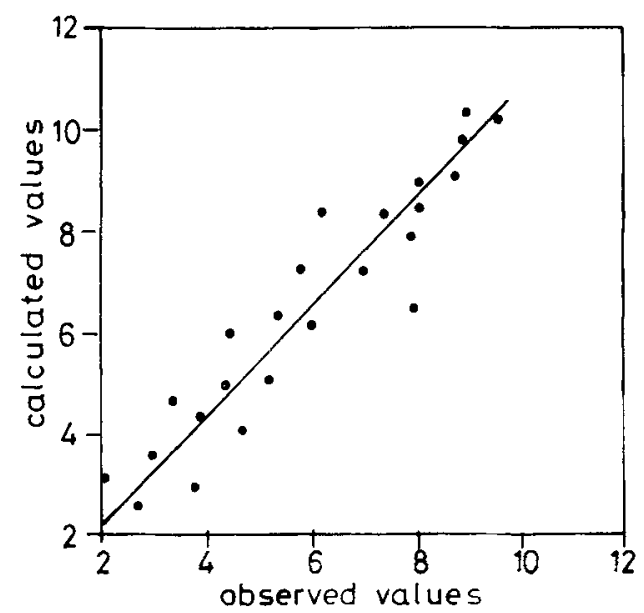

Figure 5. Calculated vs observed values for scattering coefficients at best suitable look-angle $\left(25^{\circ}\right)$ for $\mathrm{HH}-$ pol, $r^{2}=0.710, \mathrm{SE}=0.168, \mathrm{SE}$ of estimate $=1.301$. 


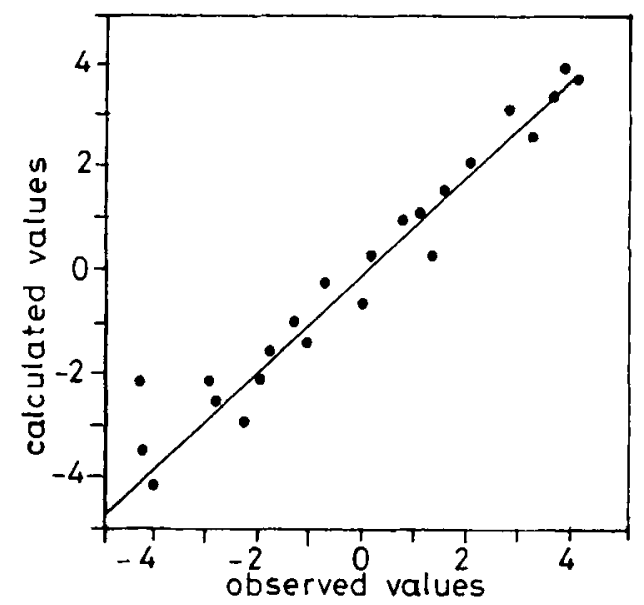

Figure 6. Calculated vs observed values for scattering coefficients at best suitable look-angle $\left(60^{\circ}\right)$ for $\mathrm{VV}$-pol, $r^{2}=0.918, \mathrm{SE}=0.108, \mathrm{SE}$ of estimate $=1.414$.

the relationship. From the results obtained and regression analysis, the following conclusions can be drawn.

(i) The scattering coefficient increases with increase in soil moisture content of the ground surface.

(ii) The best fit regression line is at look-angle $25^{\circ}, r^{2}=0.710$, standard error $=0.154$, and standard error of estimate $=1.391$ for HH-pol, whereas, for VV-pol the corresponding best look-angle $=60^{\circ}$, for $r^{2}=0.91$, standard error $=0.20$ and standard error of estimate $=1.41$.

(iii) The slope of regression is higher for larger frequency which in turn means that the higher frequency is more suitable for the study of $\sigma_{0}$ from the upper surface. This was concluded after analysing the results of both L- and C-bands (Schmugge et al 1980) and our own results of the $\mathrm{X}$-band.

(iv) It was further observed that while the VV-pol was more sensitive to soil moisture at higher look-angles, the HH-pol was more sensitive at lower look-angles. Therefore, depending on the need and the facility available, proper system parameters can be predecided for space-borne applications.

\section{References}

Bernard K, Vanclin M, Vidal-Madjar D 1981 Possible use of active microwave remote sensing data for prediction of regional evaporation by numerical simulation of soil water movement, the unsaturated zone. Water Resour. Res. 17: 1603-1610

Bertuzzi P, Chanzy A, Vidal-Madjar D, Autget M 1992 The use of microwave backscattered model for retrieving soil moisture over bare soil. Int. J. Remote Sensing 13: 2653-2668

Bradley G A, Ulaby F T 1980 Aircraft radar response to soil moisture. RSL Tech. Report 460-2, Centre for Research, University of Kansas, Lawrence, Kansas

Dobson M C, Ulaby F T 1981 Microwave backscatter dependence on surface roughness, soil moisture and soil texture: Part III - Soil tension. IEEE Trans. Geosci. Remote Sensing GE-19: 51-56

Jackson T J, O'Neill P E 1985 Aircraft scatterometer observation of soil moisture on rangeland watersheds. Int. J. Remote Sensing 6: 1135-1152 
Le Toan T, Pausader M, Flonzat G, Flutir A 1981 Soil moisture content and microwave backscatter in the 1.5-9 GHz region. Int. Geosci. Remote Sensing Symposium (IGARSS '81) Digest (IEEE)

Pervot L, Bernard R, Taconet D, Vidal-Madjar D 1984 Evaporation of bare soil evaluated using a soil water transfer model and remotely sensed surface soil moisture data. Water Resour. Res. 20: $311-316$

Schmugge T J, Jackson T J, Mckim H L 1980 Survey of methods for soil moisture determination. Water Resour. Res. 16: 961-979

Singh K P, Jha K K 1987 Effect of soil moisture and surface roughness on microwave scattering signatures. J. Wave Mater. Interaction 2: 321-334

Ulaby F T, Batlivala P P, Dobson M C 1978 Microwave backscatter dependence on surface roughness, soil moisture and soil texture: Part I - Bare soil. IEEE Trans. Geosci. Remote Sensing GE-16: 286-295

Ulaby F T, Moore R K, Fung A K 1982 Microwave remote sensing - active and passive (Norwood, MA: Artech House) vol. 2 